Audiology

Neurotology
Audiol Neurotol 2015;20:62-71

DOI: $10.1159 / 000366512$
Received: December 6, 2013

Accepted after revision: August 8, 2014 Published online: December 13, 2014

\title{
Assessment of the Effects of Superior Canal Dehiscence Location and Size on Intracochlear Sound Pressures
}

\author{
Marlien E.F. Niesten ${ }^{a-c}$ Christof Stieger ${ }^{c, e}$ Daniel J. Lee ${ }^{c} \quad J u l i e ~ P$. Merchant $^{c}$ \\ Wilko Grolman ${ }^{a, b}$ John J. Rosowski ${ }^{c, d}$ Hideko Heidi Nakajima ${ }^{c, d}$ \\ ${ }^{a}$ Department of Otorhinolaryngology, Head and Neck Surgery, University Medical Center, and b Rudolf Magnus \\ Institute of Neuroscience, Utrecht, The Netherlands; ' Department of Otology and Laryngology, Harvard Medical \\ School, Eaton Peabody Laboratory, Massachusetts Eye and Ear Infirmary, Boston, Mass., and d Speech and Hearing \\ Bioscience and Technology Program, Harvard-MIT Division of Health Sciences and Technology, Cambridge,

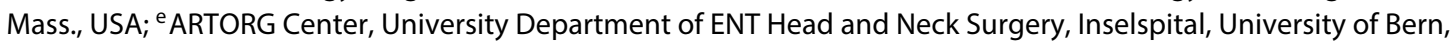 \\ Bern, Switzerland
}

\section{Key Words}

Superior semicircular canal dehiscence - Cochlear pressure . Scala vestibule $\cdot$ Scala tympani $\cdot$ Differential pressure

\begin{abstract}
Superior canal dehiscence (SCD) is a defect in the bony covering of the superior semicircular canal. Patients with SCD present with a wide range of symptoms, including hearing loss, yet it is unknown whether hearing is affected by parameters such as the location of the SCD. Our previous human cadaveric temporal bone study, utilizing intracochlear pressure measurements, generally showed that an increase in dehiscence size caused a low-frequency monotonic decrease in the cochlear drive across the partition, consistent with increased hearing loss. This previous study was limited to SCD sizes including and smaller than $2 \mathrm{~mm}$ long and 0.7 $\mathrm{mm}$ wide. However, the effects of larger SCDs ( $>2 \mathrm{~mm}$ long) were not studied, although larger SCDs are seen in many patients. Therefore, to answer the effect of parameters that have not been studied, this present study assessed the effect of SCD location and the effect of large-sized SCDs (>2 mm long) on intracochlear pressures. We used simultaneous
\end{abstract}

measurements of sound pressures in the scala vestibuli and scala tympani at the base of the cochlea to determine the sound pressure difference across the cochlear partition - a measure of the cochlear drive in a temporal bone preparation - allowing for assessment of hearing loss. We measured the cochlear drive before and after SCDs were made at different locations (e.g. closer to the ampulla of the superior semicircular canal or closer to the common crus) and for different dehiscence sizes (including larger than $2 \mathrm{~mm}$ long and $0.7 \mathrm{~mm}$ wide). Our measurements suggest the following: (1) different SCD locations result in similar cochlear drive and (2) larger SCDs produce larger decreases in cochlear drive at low frequencies. However, the effect of SCD size seems to saturate as the size increases above $2-3 \mathrm{~mm}$ long and 0.7 $\mathrm{mm}$ wide. Although the monotonic effect was generally consistent across ears, the quantitative amount of change in cochlear drive due to dehiscence size varied across ears. Additionally, the size of the dehiscence above which the effect on hearing saturated varied across ears. These findings show that the location of the SCD does not generally influence the amount of hearing loss and that SCD size can help explain some of the variability of hearing loss in patients.

(c) 2014 S. Karger AG, Base

\section{KARGER 125\%}

(C) 2014 S. Karger AG, Basel

$1420-3030 / 14 / 0201-0062 \$ 39.50 / 0$ 


\section{Introduction}

Abnormal absence of bone between the superior semicircular canal and the intracranial space, called superior canal dehiscence (SCD), can cause a range of symptoms (SCD syndrome), including hearing loss [Minor et al., 1998]. Normally, sound produces motion of the oval and round windows, allowing for the development of a pressure difference between the scala vestibuli (SV) and scala tympani (ST). This input differential pressure across the partition at the base of the cochlea - the cochlear drive is an estimate of hearing in a human temporal bone preparation [Nakajima et al., 2009]. Previously, we described a method to simultaneously measure the intracochlear sound pressures in $\mathrm{SV}$ and $\mathrm{ST}\left(\mathrm{P}_{\mathrm{SV}}\right.$ and $\left.\mathrm{P}_{\mathrm{ST}}\right)$ in order to determine the cochlear drive [Nakajima et al., 2009]. We then studied the effect of SCD on intracochlear pressures [Pisano et al., 2012], finding that SCD resulted in decreased $\mathrm{P}_{\mathrm{SV}}$ and $\mathrm{P}_{\mathrm{ST}}$ due to fluid motion shunting through an alternative low-impedance SCD pathway. Across the cochlear partition, the cochlear drive was also found to be lower with SCD, consistent with the hearing loss observed in patients with SCD [Pisano et al., 2012].

Patients with SCD syndrome present symptoms ranging from no hearing loss to a large low-frequency conductive loss. It is yet unknown whether differences in the location of the SCD affect differences in hearing. Clinically, dehiscences vary in location along the arc of the semicircular canal. Williamson et al. [2003] described that $36 \%$ of their patients had an SCD located at the arcuate eminence; the remainder had an SCD located at the posterior (36\%) or anterior (28\%) aspect of the superior semicircular canal. In addition, McCall et al. [2011] described a series of 12 patients (from a database of 131 patients) who had a more posterior-located SCD due to contact of the superior semicircular canal with the superior petrosal sinus. Furthermore, some clinical studies have found that an increase in SCD size is associated with increased hearing loss [Chien et al., 2012; Niesten et al., 2014], while others have observed no association [Chi et al., 2010; Mikulec et al., 2004]. Our previous human cadaveric temporal bone study showed results consistent with size-related hearing loss: an increase in dehiscence size (from 0.3-mm-diameter pinpoint to 2 $\mathrm{mm}$ long and $0.7 \mathrm{~mm}$ wide) caused a low-frequency monotonic decrease in the cochlear drive. Effects of larger SCDs ( $>2 \mathrm{~mm}$ long) were not assessed in our previous temporal bone studies, although many SCD patients have a dehiscence larger than $2 \mathrm{~mm}$. The computational model predictions of Songer and Rosowski [2007] indicated that the low-frequency effect of the SCD size might reach a plateau
Table 1. Summary of specimens

\begin{tabular}{lllll}
\hline $\begin{array}{l}\text { Specimen } \\
\text { No. }\end{array}$ & Comment & $\begin{array}{l}\mathrm{P}_{\text {SV }} \\
\text { reversed }\end{array}$ & $\begin{array}{l}\mathrm{P}_{\text {ST }} \\
\text { reversed }\end{array}$ & $\begin{array}{l}\mathrm{V}_{\text {Stap }} \\
\text { reversed }\end{array}$ \\
\hline $1(107)$ & Low $\mathrm{V}_{\text {Stap }}$ & & \\
\hline $2(108)$ & SCD size only & $\mathrm{x}$ & & \\
\hline $3(110)$ & Low $\mathrm{V}_{\text {Stap }}$ & & & \\
\hline $4(112)$ & & $\mathrm{x}$ & $\mathrm{x}$ & $\mathrm{x}$ \\
\hline $5(114)$ & Trauma & & & \\
\hline $6(115)$ & & $\mathrm{x}$ & & $\mathrm{x}$ \\
\hline $7(117)$ & Unstable ST sensor & & & \\
\hline $8(118)$ & & & & \\
\hline $9(122)$ & Air in cochlea & & $\mathrm{x}$ & $\mathrm{x}$ \\
\hline $10(123)$ & Low $\mathrm{V}_{\text {Stap }}$ & & $\mathrm{x}$ & $\mathrm{x}$ \\
\hline $11(124)$ & & $\mathrm{x}$ & $\mathrm{x}$ & \\
\hline $12(125)$ & & & & \\
\hline
\end{tabular}

Numbers in parentheses indicate the experiment number. $\mathrm{V}_{\text {Stap }}=$ Stapes velocity; $\mathrm{P}_{\mathrm{SV}}=$ pressure in scala vestibuli; $\mathrm{P}_{\mathrm{ST}}=$ pressure in scala tympani.

after a certain size. Furthermore, another complicating matter is that above $1 \mathrm{kHz}$, it has been observed in cadaveric preparations that a small dehiscence $(0.3-0.5 \mathrm{~mm}$ in diameter) can sometimes produce a larger decrease in cochlear drive than larger dehiscences [Pisano et al., 2012]. In this present study, we explored yet unanswered questions: we assessed the effect of SCD location and the effect of large-sized SCD on intracochlear sound pressures.

\section{Methods}

\section{Summary of Specimens}

We used 12 temporal bones from donors ranging in age from 22 to 81 years, including 6 females and 6 males (table 1). In 1 experiment, we only studied the effect of SCD size, and in all other experiments we studied the effect of the SCD location as well. From the 12 temporal bones, 6 were excluded due to complications such as low stapes velocity (due most likely to ossicular stiffness or partial disarticulation), the presence of air in the cochlea, trauma that occurred during the experiment, or sensor calibrations that differed by more than $3 \mathrm{~dB}$ in both SV and ST. Table 1 shows an overview of the 6 remaining preparations; 5 bones resulted in experiments with stable sensors in which the effects of SCD on $\mathrm{P}_{\mathrm{SV}}$ could be reversed by patching, and 3 of these 5 experiments also included reversal of the SCD effects in $\mathrm{P}_{\mathrm{ST}}$ by patching. The stapes velocity obtained in 6 temporal bones was initially normal, changed when the SCD was created, and reversed on patching. 


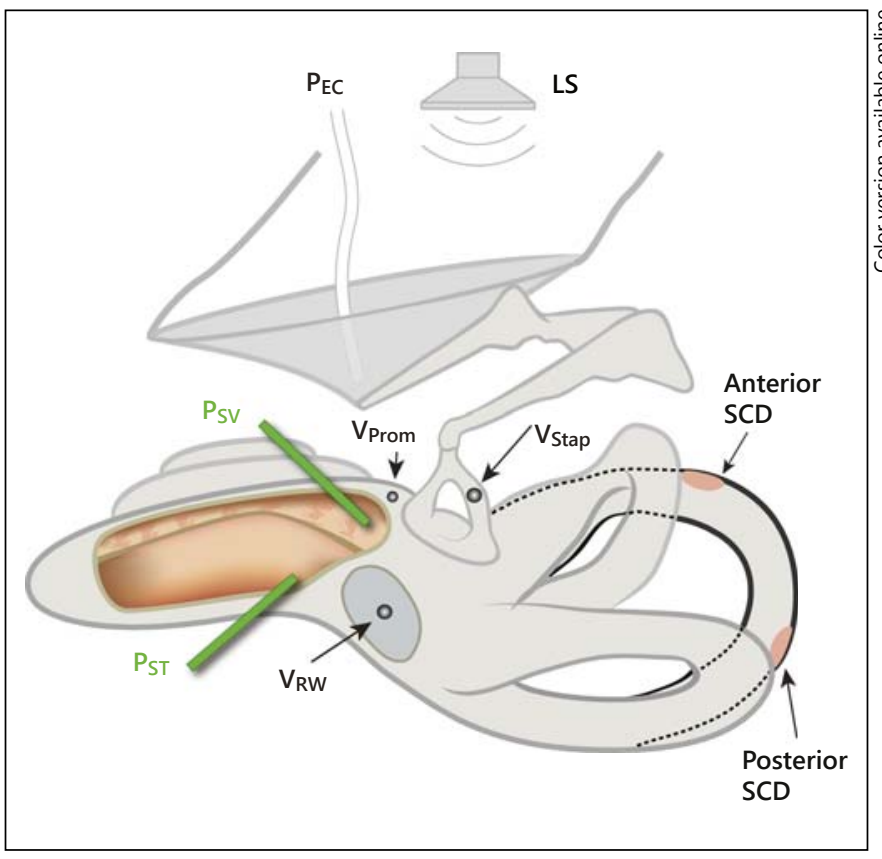

Fig. 1. Illustration of the temporal bone preparation showing the anterior and posterior SCD locations along the superior semicircular canal. $\mathrm{P}_{\mathrm{EC}}=$ Ear canal pressure; $\mathrm{V}_{\text {Prom }}$ promontory velocity; $\mathrm{V}_{\text {Stap }}=$ stapes velocity; $\mathrm{V}_{\mathrm{RW}}=$ round-window velocity; $\mathrm{LS}=$ loudspeaker. Note: the opening shown in the cochlea is for illustration purposes only.

\section{Temporal Bone Preparation}

We prepared the temporal bones as described in Nakajima et al. [2009] and Pisano et al. [2012]; therefore, only a brief description of the preparation will be given here. We used fresh human cadaveric temporal bones and previously frozen temporal bones that were removed within $24 \mathrm{~h}$ of death by the method described in Nadol [1996]. During temporal bone preparation, we opened the facial recess, thinned the area of the cochlear promontory where SV and ST sound pressure sensors would later be inserted, and removed the stapes tendon. In addition, we thinned (blue-lined) the bone overlying the superior semicircular canal along a length of $10 \mathrm{~mm}$ through the lateral transmastoid approach.

After placing a speaker at the ear canal opening and a probe tube microphone $1-3 \mathrm{~mm}$ from the umbo, the ear canal was sealed. Under fluid, we drilled 200- $\mu$ m-diameter cochleostomies in SV and ST, near the oval and round windows, into which we inserted two micro-optical sound pressure sensors of the type developed by Olson [1998]. The sensors were sealed with a dental impression material, Jeltrate (L.D. Caulk Co.), so as to prevent air from entering the inner ear and fluid from leaking out. With laser Doppler vibrometry (Polytec CLV) and reflectors on the stapes posterior crus and round-window membrane, we confirmed stability of stapes and round-window velocities before and after making cochleostomies and pressure sensor insertions. We also evaluated whether a half-cycle phase difference was present between the oval- and round-window motion in order to confirm that no air was intro-

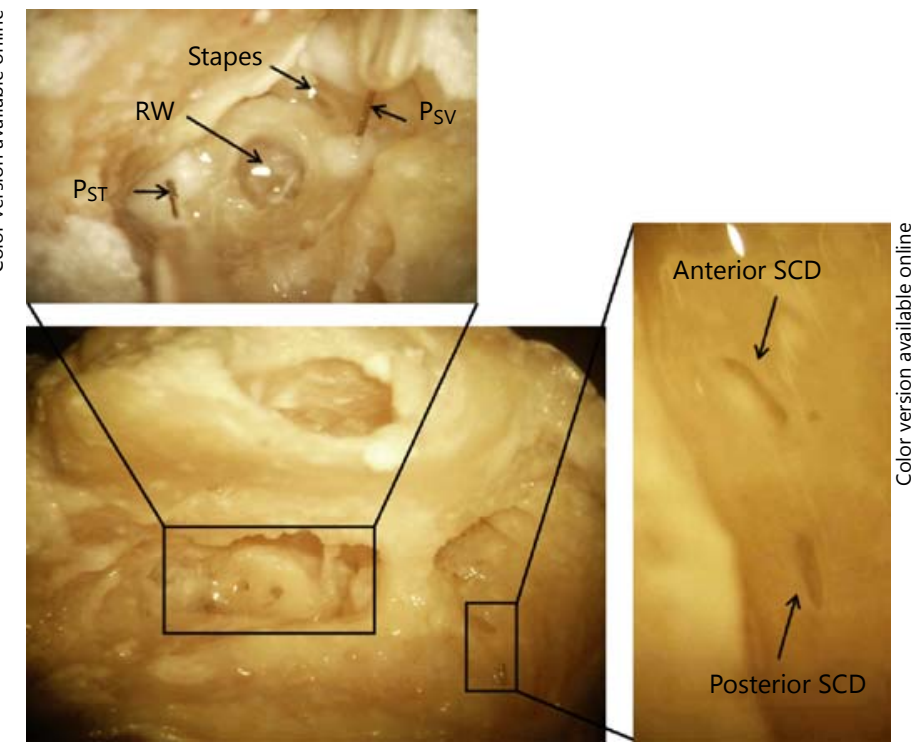

Fig. 2. Images of a temporal bone during and after an experiment. RW = Round window. The large bottom left picture shows an overview that includes the ear canal, open facial recess and exposed epitympanic space without the pressure sensors. Cochleostomies were made for the pressure sensors, and 2 dehiscences were created in the semicircular canal. The top image shows a magnified view (from a slightly different angle) to show the position of the pressure sensors inserted in SV and ST relative to the round window and stapes. The image on the right shows a magnified version of the dehiscences.

duced in the cochlea. Before and after insertion, the sensors were calibrated to ensure their stability within $3 \mathrm{~dB}$. See figure 1 for a schematic of our temporal bone preparation.

\section{Experimental Approach}

Before and after the creation of an SCD under fluid (to prevent entrance of air into the inner ear), we measured the pressures in SV, ST and the ear canal, as well as the stapes velocity for frequencies between $100 \mathrm{~Hz}$ and $10 \mathrm{kHz}$. In each temporal bone, we created dehiscences slowly with a diamond burr in two different locations: a more anterior SCD between the ampullated end and the arcuate eminence, and a more posterior SCD between the arcuate eminence and the common crus (fig. 1, 2). After each measurement with an SCD, we patched the SCD with dental impression material to ensure a reversal of the SCD effects and to confirm that the values were similar to the baseline. Then an SCD was created in the other location and measurements were made. The SCD was then patched and measurements were again made to ensure reversal to the initial state after patching the second SCD. In half of the experiments, we created the anterior SCD first, and in the other half, we created the posterior SCD first. By keeping the SCD size similar for both locations (about $1.3 \mathrm{~mm}$ long and $0.7 \mathrm{~mm}$ wide) and ensuring reversal of the SCD effects, the SCD location was therefore the only variable that changed during measurements. From our previous experiments [Pisano et al., 2012], we know that an SCD length between 1 and $1.5 \mathrm{~mm}$ shows a considerable change in pressure and that the effect is reversible with patching. 
Patching in this study was modified with respect to our earlier study [Pisano et al., 2012]. Unlike previously, where only Jeltrate was applied, we initially layered a piece of writing paper approximately the size of the dehiscence and then applied the Jeltrate over the paper; this was done to prevent Jeltrate from entering the semicircular canal. Throughout this procedure, we were careful not to introduce air into the semicircular canal.

After completing intracochlear sound pressure measurements for both SCD locations separately, we removed the Jeltrate and paper from the SCDs and measured the intracochlear pressures with both SCDs open. It was noted that upon removal of the paper and Jeltrate, the contents of the lumen did not appear traumatized, as it is likely that the Jeltrate did not obstruct the lumen. In addition, we drilled away the bone between the anterior and posterior SCDs to create one large dehiscence of around $4 \mathrm{~mm}$ long and $0.7 \mathrm{~mm}$ wide, which is approximately the median SCD size found in a study assessing SCD size and location in 104 patients clinically [Niesten et al., 2014]. We patched the different SCDs after each measurement to ensure reversal of the effects. Figure 2 shows photographs of the preparation, with sensors inserted in the cochlea and an anterior and a posterior dehiscence in the superior semicircular canal.

\section{Results}

\section{Stapes Velocity}

In all 6 temporal bones, the velocity of the stapes changed when an SCD was made, and reversed back to the initial value after the dehiscence was patched (similar to Pisano et al., [2012]). In all but 1 experiment, the stapes velocity increased at varying frequencies after creating a dehiscence. This has been explained by the idea of the fluid motion shunting through the SCD, allowing for larger movement of the stapes [Chien et al., 2007; Pisano et al., 2012]. However, in 1 experiment, the stapes velocity did the opposite: it decreased following SCD and reversed back to its initial velocity following patching. These results were similar to those found in Pisano et al. [2012], where it was found and described that the changes in stapes velocity due to SCD varied across ears and was unrelated to the cochlear input drive. The impedance at the oval window (influencing stapes velocity) is probably influenced by relative impedances of various structures in the inner ear to the impedance of the annular ligament between the footplate and cochlea. Therefore, stapes velocity serves as a poor indicator of the effects of SCD on hearing.

\section{Effects of SCD Location}

SV Pressure

Overall, 4 experiments had stable sensors in SV and reversal of the SCD effects after patching. In 2 experiments we created the anterior SCD first, and in the 2 oth- ers we created the posterior SCD first. The first SCD was patched before the second SCD was created, with confirmation of reversals in pressures to the initial values; therefore, results were not influenced by which SCD location was created first (anterior or posterior). In each ear, the anterior and posterior SCDs were about the same size $(0.7 \mathrm{~mm}$ wide) and approximately the same length (within $0.2 \mathrm{~mm}$ ). Across ears, the length varied between 1.2 and $1.5 \mathrm{~mm}$ long.

Figure 3 illustrates a representative example showing a similar drop in $\mathrm{P}_{\mathrm{SV}}$ for the anterior and posterior SCD. For each specimen, similar decreases in $\mathrm{P}_{\mathrm{SV}}$ resulted with anterior and posterior SCD, (within $0-4 \mathrm{~dB}$, except for experiment 112 shown in figure 3 where the lowest frequency of $100 \mathrm{~Hz}$ had a difference of $6.8 \mathrm{~dB}$ ). However, as shown in figure 4 , the magnitude of change in pressure due to SCD varied among the 4 temporal bones, ranging from 8 to $18 \mathrm{~dB}$, similar to variations seen across bones in Pisano et al. [2012]. The effects of SCD were mainly present in the low frequencies - up to $1 \mathrm{kHz}$. In the mid-to-high frequencies $(1-2 \mathrm{kHz})$ we either found a slight increase or decrease in $\mathrm{P}_{\mathrm{SV}}$, or we did not see an effect. In 1 temporal bone, we found a slight increase in $\mathrm{P}_{\mathrm{SV}}$ between 1 and $2 \mathrm{kHz}$.

\section{ST Pressure}

We had 3 temporal bones with stable sensors in ST and reversal of the SCD effects after patching. Figure 3 shows an example of $\mathrm{P}_{\mathrm{ST}}$ before and after anterior and posterior SCDs were made for a representative experiment. The drop in $\mathrm{P}_{\mathrm{ST}}$ was similar for both SCD locations, ranging from 0 to $4 \mathrm{~dB}$ within each temporal bone (except for experiment 112: at $100 \mathrm{~Hz}$ there was a difference of $6.9 \mathrm{~dB}$ between the anterior and posterior SCD). Similar to Pisano et al. [2012], the change in $\mathrm{P}_{\mathrm{ST}}$ across temporal bones varied and ranged from 8 to $17 \mathrm{~dB}$, as shown in figure 4 . The effects were mainly present up to $600-800 \mathrm{~Hz}$. In the high frequencies, $\mathrm{P}_{\mathrm{ST}}$ remained similar or changed slightly, comparable to Pisano et al. [2012]. For 1 experiment (experiment 125), the measurements were close to the noise level at high frequencies.

\section{Cochlear Drive}

Overall, 3 experiments had stable sensors and reversal of the SCD effects on both SV and ST pressures, for which we were able to calculate differential pressure across the partition at the base of the cochlea $(\Delta \mathrm{P})$. Figure 5 shows two examples of $\Delta \mathrm{P}$. In addition to the low-frequency decrease in $\mathrm{P}_{\mathrm{SV}}$ and $\mathrm{P}_{\mathrm{ST}}$, we found a decrease in $\Delta \mathrm{P}$ after the creation of an SCD. We found a similar low-frequency decrease in differential pressure for the anterior and 


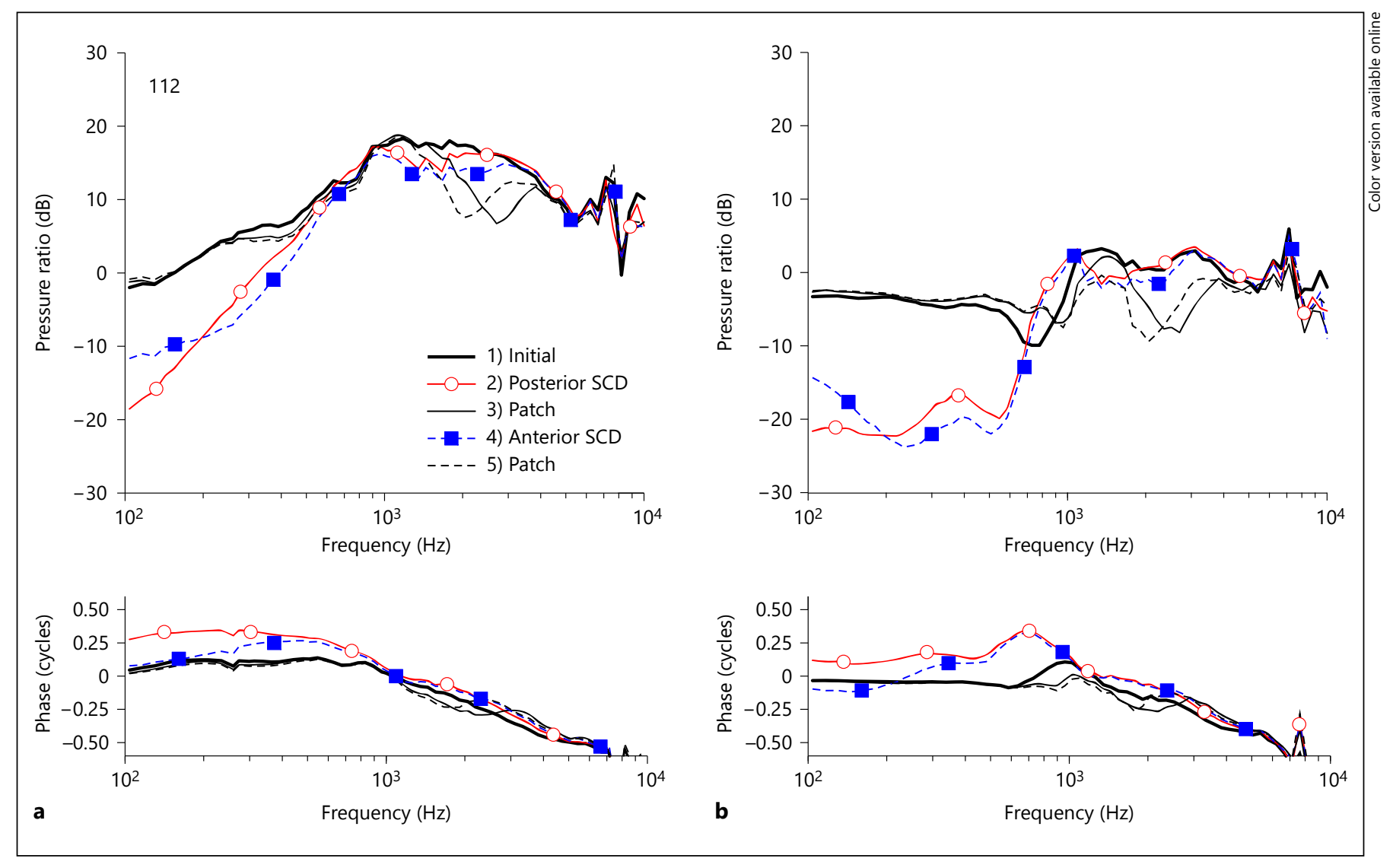

Fig. 3. Effect of dehiscence location. $\mathrm{P}_{\mathrm{EC}}=$ Ear canal pressure. $\mathrm{P}_{\mathrm{SV}}$ normalized to ear canal pressure $\left(\mathrm{P}_{\mathrm{SV}} / \mathrm{P}_{\mathrm{EC}}, \mathbf{a}\right)$ and $\mathrm{P}_{\mathrm{ST}}$ normalized to ear canal pressure $\left(\mathrm{P}_{\mathrm{ST}} / \mathrm{P}_{\mathrm{EC}}, \mathbf{b}\right)$, before and after the creation of an SCD in 2 different locations. The black thick solid line shows the initial intracochlear sound pressure; the solid line with circles shows the lower pressure after the creation of the posterior SCD.

posterior SCD, indicating that the location of the SCD does not influence the differential pressure. This would correspond clinically with similar hearing loss for patients with an SCD located either more anteriorly or more posteriorly. The low-frequency decrease in $\Delta \mathrm{P}$ ranged from $10-20 \mathrm{~dB}$ up to $600-800 \mathrm{~Hz}$. Since the magnitude of $\mathrm{P}_{\mathrm{SV}}$ was generally larger than $\mathrm{P}_{\mathrm{ST}}$, the cochlear drive is mostly influenced by $\mathrm{P}_{\mathrm{SV}}$ changes [Stieger et al., 2013] and can be appreciated by comparing experiment 112 in figures 3 and 5.

\section{Effects of Larger SCD Size}

\section{SV Pressure}

In 4 temporal bones we had stable sensors and a reversal of SCD effects on $\mathrm{P}_{S V}$ for various SCD sizes. Figure 6 shows a representative example of the different SCD sizes, starting with an SCD length of $0.6 \mathrm{~mm}$, a larger SCD of

The SCD was then patched to ensure reversal of the pressures, shown by the thin solid black lines. Then, an anterior SCD was created, resulting in the lower pressures shown by the dotted line with squares. At the end of the experiment the SCD is patched, as indicated by the thin dashed black line.

$1.2 \mathrm{~mm}$, then one of $2.4 \mathrm{~mm}$, and finally an SCD length of $3.0 \mathrm{~mm}$. The width was around $0.5 \mathrm{~mm}$ for the small SCDs and around $0.7 \mathrm{~mm}$ for the larger SCDs. In the low frequencies, and primarily at $100 \mathrm{~Hz}$, we found a larger decrease in $\mathrm{P}_{\mathrm{SV}}$ when the dehiscence size increased. As illustrated in figure 6 , the smaller SCD of $0.6 \mathrm{~mm}$ seemed to cause less decrease in pressure up to $1 \mathrm{kHz}$, which is consistent with data from Pisano et al. [2012]. Above $200-300 \mathrm{~Hz}$, not much variation existed in the amount of pressure drop for the larger SCDs $(1.2-3.0 \mathrm{~mm})$. Generally, $\mathrm{P}_{\mathrm{SV}}$ decreased monotonically when the SCD size increased, ranging from 2 to $8 \mathrm{~dB}$ within each temporal bone. The effect of SCD size was more prominent for smaller SCDs $(<1.5 \mathrm{~mm})$ and seemed to saturate as the size increased above approximately $2-4 \mathrm{~mm}$ long and $0.7 \mathrm{~mm}$ wide (with the size for which saturation of SCD effects occurred varying across ears). Because large dehis- 


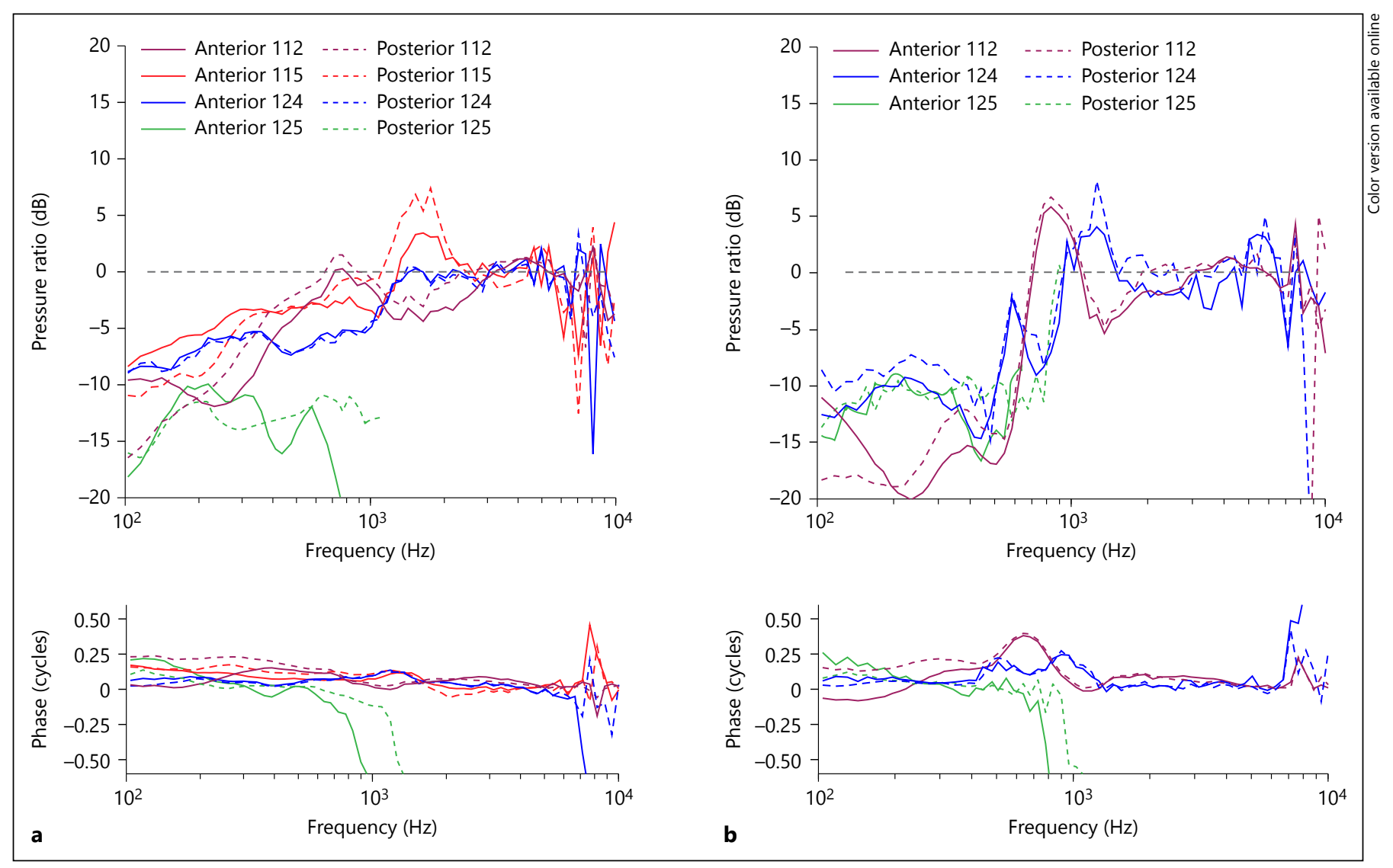

Fig. 4. Overview effect of SCD location. Change in $P_{S V}$ (vs. initial) for 4 temporal bones (a) and in $\mathrm{P}_{\mathrm{ST}}$ (vs. initial) for 3 temporal bones (b). The sizes for both locations were similar (112: anterior

cence sizes ( $>2 \mathrm{~mm}$ length) were studied in this article, compared to Pisano et al. [2012] where lengths $<2 \mathrm{~mm}$ were studied, we were able to determine that the size effect saturated. In the high frequencies, the intracochlear sound pressures were similar or slightly decreased due to the dehiscence. This amount of decrease in pressure was variable across different temporal bones.

\section{ST Pressure}

We had 2 bones with successful reversal of SCD effects in $\mathrm{P}_{\mathrm{ST}}$ due to different SCD sizes. Figure 6 shows a representative example of $\mathrm{P}_{\mathrm{ST}}$, where we made intracochlear sound pressure measurements for SCD lengths of 1.3, 2.7 and $4.5 \mathrm{~mm}$. The figure illustrates that pressure decreased with an increase in SCD size - up to 500-600 Hz. Generally, the $\mathrm{P}_{\mathrm{ST}}$ decreased further as SCD size increased, ranging in pressure drop from 2 to $7 \mathrm{~dB}$ within each temporal bone. The effects of SCD size on $\mathrm{P}_{\mathrm{ST}}$ were generally similar to those in SV in that after an increase in dehis-

SCD Location and Size on Intracochlear Pressures
$1.3 \mathrm{~mm}$ and posterior $1.4 \mathrm{~mm}$; 115: anterior $1.35 \mathrm{~mm}$ and posterior $1.5 \mathrm{~mm}$; 124: anterior and posterior $1.2 \mathrm{~mm}$, and 125: anterior and posterior $1.4 \mathrm{~mm}$ ).

cence length of over 1.5-3 mm the incremental decrease in $\mathrm{P}_{\mathrm{ST}}$ was less pronounced, though saturation was not always reached (fig. 6). Magnitude variation of the pressure drop across ears existed for $\mathrm{P}_{\mathrm{ST}}$ as well.

\section{Cochlear Drive}

Pisano et al. [2012] showed that $\Delta \mathrm{P}$ decreased as the SCD size increased up to around $2 \mathrm{~mm}$. Magnitude of $\Delta \mathrm{P}$ depended more on $\mathrm{P}_{\mathrm{SV}}$ than on $\mathrm{P}_{\mathrm{ST}}$, because $\mathrm{P}_{\mathrm{SV}}$ was generally much larger in magnitude than $\mathrm{P}_{\mathrm{ST}}$, that is, $\Delta \mathrm{P}=$ $\left(\mathrm{P}_{\mathrm{SV}}-\mathrm{P}_{\mathrm{ST}}\right) \approx \mathrm{P}_{\mathrm{SV}}$ (see explanation and illustration of vector representation of intracochlear pressures in Stieger et al. [2013]). Reversal of the effects in both scalae became more challenging as the SCD size increased, especially for SCDs around 3-4 $\mathrm{mm}$. While we did not have an experiment with perfect reversal of both $\mathrm{P}_{\mathrm{SV}}$ and $\mathrm{P}_{\mathrm{ST}}$ after large SCDs of $4 \mathrm{~mm}, \triangle \mathrm{P}$ would decrease for larger SCD sizes, similar to the change in $\mathrm{P}_{\mathrm{SV}}$, and it is likely that this effect would reach saturation as the SCD size reached a critical point. 


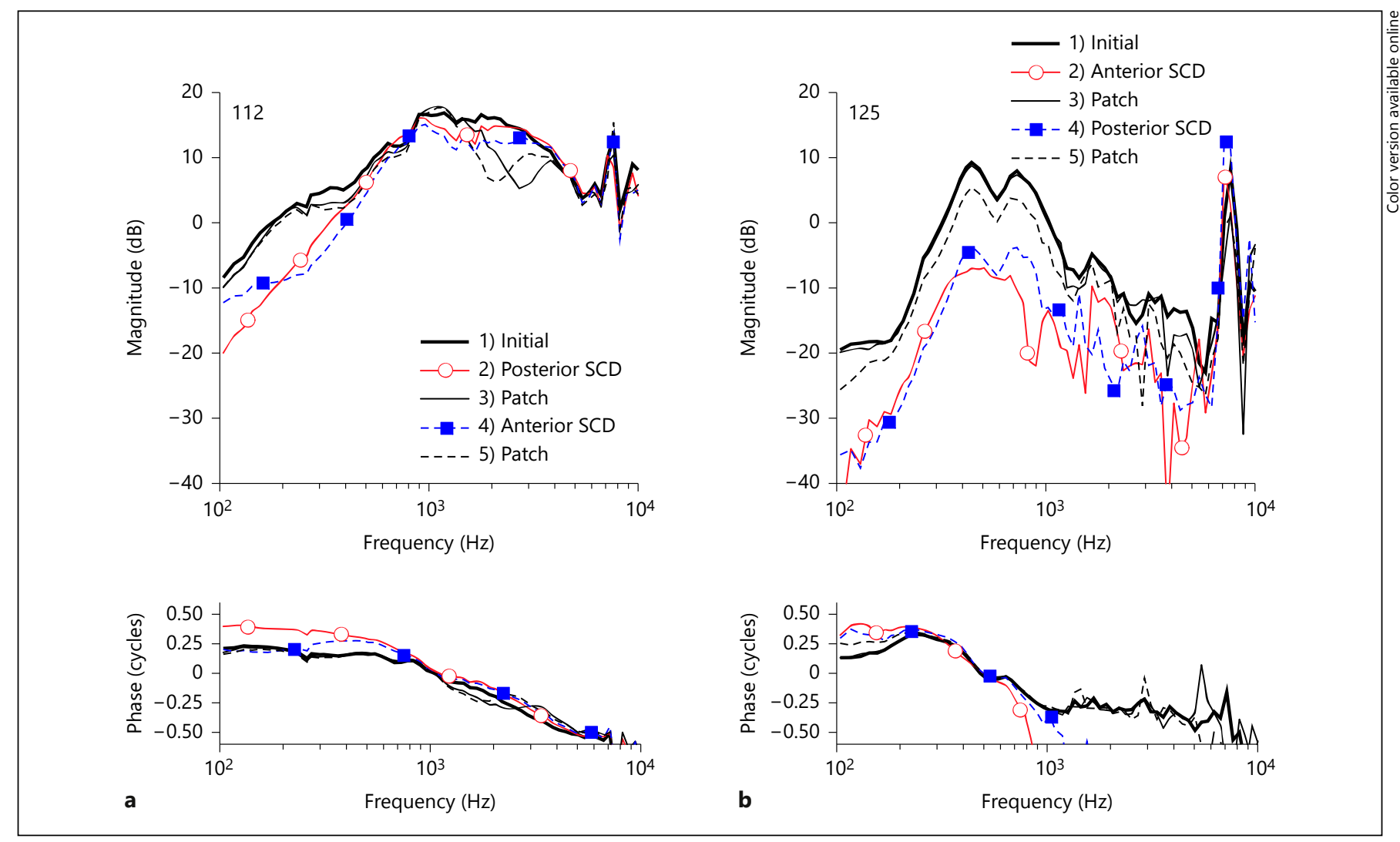

Fig. 5. Illustration of 2 representative experiments (a and $\mathbf{b}$ ). Differential pressure before and after the creation of an anterior and posterior SCD. The black solid line shows the initial intracochlear sound pressure; the solid line with circles shows the decrease in pressure after creation of the first SCD. The SCD was then patched to ensure reversal of the pressures, shown by the thin solid black line. The dotted line with squares shows the drop in pressure after the second SCD was created. At the end of the experiment, the second SCD was patched, as indicated by the thin dashed black line.

\section{Discussion}

Our measurements suggest the following: (1) differences in SCD location do not affect changes seen in intracochlear pressures and (2) larger SCDs produce larger decreases in cochlear drive at low frequencies, but this effect seems to saturate as size increases above 2-4 $\mathrm{mm}$ long and $0.7 \mathrm{~mm}$ wide (though varying across ears). The results of this present study for small dehiscences are consistent with those of Pisano et al. [2012]. However, in Pisano et al., the location of the dehiscence was held constant and the sizes were less than $2 \mathrm{~mm}$ long. In this study, we were able to show the saturation of the effect of SCD for dehiscences greater than approximately $2 \mathrm{~mm}$. The magnitude of pressure change for similarly sized SCDs varied across ears as well as the size of dehiscence to result in the saturation of the SCD effects.

\section{Effect of SCD Location}

To study the effect of SCD location, all variables (such as the length, shape and width of the SCD) were kept constant and only variations in SCD location were made. Different SCD locations resulted in similar decreases in lowfrequency pressures, as shown in figure 4 . In 2 experiments (experiments 124 and 125), each ear had anterior and posterior SCDs of the same length, resulting in the same decrease (within $2 \mathrm{~dB}$ ) in cochlear drive. In 2 other experiments, there was a slight difference in SCD length (within $0.2 \mathrm{~mm}$ ) between the two locations, resulting in a slight difference in the decreases of pressures (within $5 \mathrm{~dB}$ ).

When we compare our outcomes with our clinical study that assessed the effect of SCD size and location on hearing, we find similar results. The clinical study also showed that the location of the SCD did not correlate with the amount of hearing loss [Niesten et al., 2014].
68
Niesten/Stieger/Lee/Merchant/Grolman/ Rosowski/Nakajima 


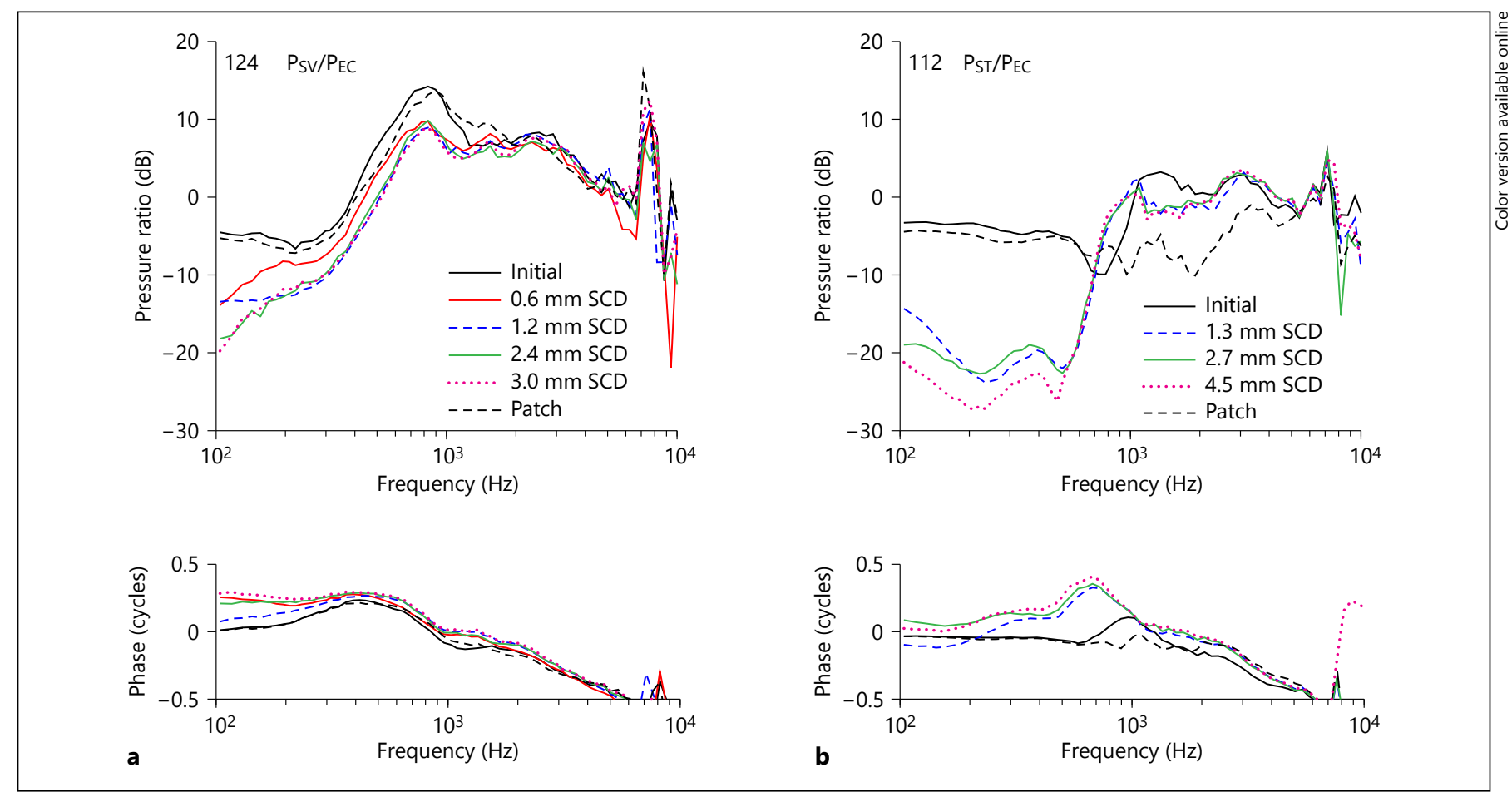

Fig. 6. Effect of dehiscence size. $\mathrm{P}_{\mathrm{SV}} / \mathrm{P}_{\mathrm{EC}}(\mathbf{a})$ and in $\mathrm{P}_{\mathrm{ST}} / \mathrm{P}_{\mathrm{EC}}(\mathbf{b})$, before and after the creation of different sizes of SCD in 2 different experiments. The black solid line shows the initial intracochlear sound pressure; the other lines show the pressure after different
SCD sizes are created. Between the measurements, the SCD was patched to ensure reversal of the pressures, and at the end of the experiment, the SCD was patched, as indicated by the thin dashed black line.
Predictions in an animal model hypothesized that an SCD closer to the ampulla would show slightly more effect on hearing than an SCD further away from the ampulla, but that the SCD location could not account for large differences in hearing [Rosowski et al., 2004]. It could be theorized that the fluid flow may divide preferentially towards the ampullated end of the semicircular canal or to the common crus, depending on where the dehiscence is located, and therefore diversion of fluids through a dehiscence located more medially/posteriorly may be just as easy as through a dehiscence located more anteriorly/laterally. To clarify this mechanism, and to investigate the direction of fluid flow when an SCD is created, sound pressure measurements in the semicircular canal itself might help to address this question in the future.

\section{Effect of SCD Size}

In this study, we found that when all factors were kept similar and only variations in the SCD size were made, a larger SCD caused a larger drop in pressure, but that this effect seemed to saturate as the SCD size increased be- yond a critical point (approximately $2 \mathrm{~mm}$ in length). A theory that might explain this phenomenon is that increasing SCD size results in lowered impedance through the SCD, but at some point other limits dominate and further increase in size does not appreciably continue to lower the inner-ear impedance. Our results correspond with an animal computational modeling study predicting that the effect of SCD size will not continue to influence hearing outcomes after a certain size is reached [Songer and Rosowski, 2007]. The authors proposed that the magnitude of hearing loss is affected by the increase in dehiscence size, but that this loss is maximal once the SCD size exceeds the diameter of the canal in the chinchilla. However, our human temporal bone experiments showed that the length of SCD to result in saturation was approximately twice the diameter of the semicircular canal.

Some clinical studies report that a larger SCD tends to correlate with a larger air-bone gap [Chien et al., 2012; Niesten et al., 2014; Yuen et al., 2009] or that no correlation between SCD size and hearing loss exists [Chi et al., 2010; Martin et al., 2009; Mikulec et al., 2004]. The large variation in cochlear pressure drop across the different 
ears for similar-sized SCDs in temporal bone preparations might help explain the results from these clinical studies, and the number of patients in some of these clinical studies might also have been too small to study the effect of SCD size on hearing due to this large variability among ears. Furthermore, in some ears, cochlear pressures in temporal bones were affected even more by very small SCDs than by large SCDs at frequencies above $1 \mathrm{kHz}$, also complicating the effects that SCD size has on inner ear pressures, hearing, balance, and other symptoms [Pisano et al., 2012].

\section{Limitations of the Study}

It has been shown that differential pressure $\Delta \mathrm{P}$ can be used to make an estimate of hearing in a human cadaveric temporal bone preparation (assuming the neurosensory system is intact) because it estimates the input drive across the partition despite changes in cochlear impedances. It is a good method to assess the effect of one variable in a controlled setting by keeping the other variables constant within the same ear by ensuring that effects are reversible. However, although this method provides insight into hearing in live humans, it is still difficult to determine the influences of factors that are different in the clinical situation.

Isolated temporal bone preparations do not include soft tissues such as the dura and brain above the superior semicircular canal. In some cases, the dura and brain could seal or plug the SCD. It is possible that natural plugging by the dura and brain can prevent symptoms despite having anatomical SCD diagnosed by CT [Brandolini and Modugno, 2012]. Natural plugging or patching by soft tissue could also explain why many patients report a 'second event', a trauma, 'unmasking' SCD symptoms, and why patients present with a myriad of signs and symptoms. Adding pressure at the dehiscence with a column of fluid, as well as patching the dehiscence with dura, has been shown to result in a decreased mechanical effect of SCD [Luers et al., 2014]. In our experiments we found that resurfacing the SCD with dental cement (which dries to a hard substance, but requires a dry surface to set well) often does not reverse the effects of SCD, probably due to a persistent small leak. However, resurfacing with dental impression material (Jeltrate, which dries to a soft material, but can set in a wet environment) usually reverses the effect of SCD, thus producing a tight water seal for the dehiscence. In the future, studies with whole head specimens could investigate the effect of the dura and brain on SCD [Stieger et al., 2012].
In some experiments with stable sensors, we could not achieve full reversal of the effects of a large SCD after patching the dehiscence. Patching a larger dehiscence was more challenging than patching a smaller dehiscence (up to $2 \mathrm{~mm}$ ). We also found that with our method of 'resurfacing' the dehiscence with paper and dental impression material, slight leakage of the dental impression material into the superior canal sometimes occurred. Still, in most cases, the resurfacing material was easily removed in one piece without notable trauma to the lumen of the canal.

\section{Clinical Implication and Future Directions}

We found an effect of SCD on cochlear pressure predominantly in the low frequencies, but the magnitude of the effect varied among different ears. At low frequencies near $100 \mathrm{~Hz}$, some specimens showed a large effect, while others showed only a small decrease in pressure at this frequency. The frequency range in which the effect of an SCD is visible seems to vary among ears. This could help explain the variation among patients in conductive hearing loss across the different frequencies. We also suspect the effect of SCD to be larger in frequencies below $100 \mathrm{~Hz}$, and we plan to assess this in our future studies. In addition, in patients with SCD syndrome, bone conduction thresholds are decreased [Mikulec et al., 2004; Minor et al., 2003], and similar results have been found in an animal model studying SCD [Rosowski et al., 2004; Songer and Rosowski, 2007]. We plan to study the effect of SCD on bone conduction in our temporal bone model in the future.

\section{Conclusions}

The location of the dehiscence in the superior canal does not seem to affect the severity of hearing loss. However, the large clinical variation in hearing loss can be partly explained by a difference in SCD size: a larger SCD seems to correspond with a larger drop in pressure within each ear below $1 \mathrm{kHz}$, but this effect seems to saturate as the SCD size increases. Nevertheless, across ears, the magnitude of the SCD effect appears to vary.

\section{Acknowledgments}

This study was funded by the NIH/NIDCD (grant R03DC011158-03). The authors wish to thank Diane Jones, Mike Ravicz and Melissa McKinnon.
Niesten/Stieger/Lee/Merchant/Grolman/ Rosowski/Nakajima 


\section{References}

Brandolini C, Modugno GC: Do signs of natural plugging of superior semicircular canal dehiscence exist? Am J Otolaryngol 2012;33:268271.

Chi FL, Ren DD, Dai CF: Variety of audiologic manifestations in patients with superior semicircular canal dehiscence. Otol Neurotol 2010;31:2-10.

-Chien W, Janky KL, Minor LB, Carey J: Superior canal dehiscence size: multivariate assessment of clinical impact. Otol Neurotol 2012; 33:810-815.

-Chien W, Ravicz ME, Rosowski JJ, Merchant SN: Measurements of human middle- and innerear mechanics with dehiscence of the superior semicircular canal. Otol Neurotol 2007;28: 250-257.

-Luers JC, Pazen D, Meister H, Lauxmann M, Eiber A, Beutner D, Huttenbrink KB: Acoustic effects of a superior semicircular canal dehiscence: a temporal bone study. Eur Arch Otorhinolaryngol 2014, Epub ahead of print.

- Martin C, Chahine P, Veyret C, Richard C, Prades JM, Pouget JF: Prospective radiological study concerning a series of patients suffering from conductive or mixed hearing loss due to superior semicircular canal dehiscence. Eur Arch Otorhinolaryngol 2009;266:1175-1181.

- McCall AA, McKenna MJ, Merchant SN, Curtin HD, Lee DJ: Superior canal dehiscence syndrome associated with the superior petrosal sinus in pediatric and adult patients. Otol Neurotol 2011;32:1312-1319.
Mikulec AA, McKenna MJ, Ramsey MJ, Rosowski JJ, Herrmann BS, Rauch SD, Curtin HD, Merchant SN: Superior semicircular canal dehiscence presenting as conductive hearing loss without vertigo. Otol Neurotol 2004;25: 121-129.

Minor LB, Carey JP, Cremer PD, Lustig LR, Streubel SO, Ruckenstein MJ: Dehiscence of bone overlying the superior canal as a cause of apparent conductive hearing loss. Otol Neurotol 2003;24:270-278.

-Minor LB, Solomon D, Zinreich JS, Zee DS: Sound- and/or pressure-induced vertigo due to bone dehiscence of the superior semicircular canal. Arch Otolaryngol Head Neck Surg 1998;124:249-258.

Nadol JB Jr: Techniques for human temporal bone removal: information for the scientific community. Otolaryngol Head Neck Surg 1996;115:298-305.

Nakajima HH, Dong W, Olson ES, Merchant SN, Ravicz ME, Rosowski JJ: Differential intracochlear sound pressure measurements in normal human temporal bones. J Assoc Res Otolaryngol 2009;10:23-36.

Niesten MEF, Hamberg L, Silverman J, Lou KV, McCall AA, Windsor A, Curtin HD, Herrmann BS, Grolman W, Nakajima HH, Lee DJ: Association of size and location of superior canal dehiscence with clinical presentation, audiometric and vestibular testing. Audiol Neurotol 2014;19:97-105.

Olson ES: Observing middle and inner ear mechanics with novel intracochlear pressure sensors. J Acoust Soc Am 1998; 103:34453463.
Pisano DV, Niesten ME, Merchant SN, Nakajima $\mathrm{HH}$ : The effect of superior semicircular canal dehiscence on intracochlear sound pressures. Audiol Neurootol 2012;17:338-348.

Rosowski JJ, Songer JE, Nakajima HH, Brinsko KM, Merchant SN: Clinical, experimental, and theoretical investigations of the effect of superior semicircular canal dehiscence on hearing mechanisms. Otol Neurotol 2004;25: 323-332.

-Songer JE, Rosowski JJ: A mechano-acoustic model of the effect of superior canal dehiscence on hearing in chinchilla. J Acoust Soc Am 2007;122:943-951.

- Stieger C, Candreia C, Kompis M, Herrmann G, Pfiffner F, Widmer D, Arnold A: Laser Doppler vibrometric assessment of middle ear motion in Thiel-embalmed heads. Otol Neurotol 2012;33:311-318.

-Stieger C, Rosowski JJ, Nakajima HH: Comparison of forward (ear-canal) and reverse (round-window) sound stimulation of the cochlea. Hear Res 2013;301:105-114.

Williamson RA, Vrabec JT, Coker NJ, Sandlin M: Coronal computed tomography prevalence of superior semicircular canal dehiscence. Otolaryngol Head Neck Surg 2003;129:481489.

Yuen HW, Boeddinghaus R, Eikelboom RH, Atlas MD: The relationship between the airbone gap and the size of superior semicircular canal dehiscence. Otolaryngol Head Neck Surg 2009;141:689-694.
SCD Location and Size on Intracochlear Pressures
Audiol Neurotol 2015;20:62-71 DOI: $10.1159 / 000366512$ 